\title{
Factors associated with SARS-CoV-2 antibody titers and prognosis of breakthrough infection in hemodialysis patients
}

\author{
Masataro Toda ${ }^{1} \cdot$ Ayumi Yoshifuji ${ }^{1}$ (C) Kan Kikuchi ${ }^{2} \cdot$ Masayoshi Koinuma $^{3} \cdot$ Motoaki Komatsu $^{1} \cdot$ Kentaro Fujii $^{1}$. \\ Ai Kato ${ }^{1} \cdot$ Takahide Kikuchi $^{4}$ - Atsushi Nakazawa ${ }^{5} \cdot$ Munekazu Ryuzaki $^{1}$
}

Received: 21 October 2021 / Accepted: 23 January 2022 / Published online: 8 February 2022

(C) The Author(s), under exclusive licence to The Japanese Society of Nephrology 2022, corrected publication 2022

\begin{abstract}
Background The mortality rate of Coronavirus disease 2019 (COVID-19) is extremely high in hemodialysis patients (HDP). These patients also develop lower antibody titers after vaccination. Therefore, factors associated with antibody titers and vaccine efficacy in HDP with breakthrough infection need to be investigated.

Methods We measured anti-S1 antibody titers in HDP $(n=104)$ and controls $(n=35)$, evaluating the influence of background on HDP by multivariable regression analysis. We classified 26 HDP patients admitted with COVID-19 into the unvaccinated $(n=15)$ and breakthrough infection group $(n=11)$, performing between-group comparisons of laboratory findings and prognosis. Vaccinated COVID-19 patients were classified into HDP and non-HDP controls, and compared the relationship between antibody titer and severity, and the prognosis of breakthrough infection.

Results The antibody titer was significantly lower in the HDP group than in the control group. Among HDP, age and smoking history were significantly independent factors associated with antibody titer. The breakthrough infection group had significantly better laboratory findings (KL-6 and LDH), severity, and hospitalization period than the unvaccinated group even if antibody titers were lower than the known threshold for neutralization $(p<0.05)$. There was no significant difference in prognosis between the HDP and non-HDP with breakthrough infection. Severity of COVID-19 tended to be higher with lower antibody titer in non-HDP, but not in HDP.

Conclusion Vaccines improved the severity of COVID-19 and hospitalization period of breakthrough infection in HDP, although HDP, especially in elderly smokers had lower antibody titers than control. There was no significant association between antibody titer and severity in HDP.
\end{abstract}

Keywords COVID-19 $\cdot$ Hemodialysis $\cdot$ Vaccine $\cdot$ Antibody titer $\cdot$ Breakthrough infection

Ayumi Yoshifuji

ayutsuranaide@hotmail.com

1 Department of Nephrology, Tokyo Saiseikai Central Hospital, 1-4-17 Mita, Minato-ku, Tokyo 108-0073, Japan

2 Division of Nephrology, Shimoochiai Clinic, Tokyo, Japan

3 Faculty of Pharmaceutical Sciences, Teikyo Heisei University, Tokyo, Japan

4 Department of Hematology, Tokyo Saiseikai Central Hospital, Tokyo, Japan

5 Department of Gastroenterology, Tokyo Saiseikai Central Hospital, Tokyo, Japan

\section{Introduction}

Coronavirus disease 2019 (COVID-19), caused by infection with severe acute respiratory syndrome coronavirus 2 (SARS-CoV-2) has been declared a pandemic by the World Health Organization (WHO), infecting more than tens of millions of people worldwide [1]. Hemodialysis (HD) patients are at a significantly higher risk of COVID-19 than the general population (5-16 times higher) with a mortality rate of nearly $20 \%$ [2]. Currently, since there are few drugs to effectively treat COVID-19, and HD patients are at particularly higher risk, major nephrology societies have recommended priority vaccination for HD patients [3]. To assess humoral immune responses after vaccination, quantitative assay for immunoglobulin $\mathrm{G}$ ( $\mathrm{IgG}$ ) antibodies to spike protein of SARS-CoV-2 is widely used, because anti-spike IgG 
antibody titers strongly correlated with neutralization $[4,5]$. Since HD patients have difficulty developing and maintaining sufficient humoral immunity from vaccination against influenza or hepatitis B, it is believed that HD patients may not develop and maintain sufficient humoral immunity from COVID-19 vaccination [6]. Therefore, it is important to investigate what kinds of factors affect the antibody titers in HD patients.

The COVID-19 vaccine has become widespread and has been shown to be effective in preventing COVID-19. [1] However, some cases of "breakthrough infection", which was defined as COVID-19 infection more than 14 days after full vaccination, have been reported [5]. Although higher anti-spike IgG antibody titers were associated with a lower risk of breakthrough infection and lower viral RNA copy numbers in breakthrough cases, HD patients may not be able to develop and maintain a sufficient antibody titer [5]. Therefore, the relationship between antibody titer and the efficacy of the vaccine in preventing severe complications after COVID-19 infections in HD patients needs to be investigated.

\section{Materials and methods}

\section{SARS-CoV-2 antibody titer in the HD and control groups after vaccination}

We measured IgG antibody titers to $\mathrm{S} 1$ subunit of spike protein of SARS-CoV-2 (anti-S1 antibody titers) by Abbott SARS-CoV-2 IgG II Quantitative Antibody Assay in 104 HD patients (HD group) and 35 hospital staff (non-HD control group) who belong to Shimoochiai Clinic one month after two doses of the BNT162b2 mRNA COVID-19, performing between-group comparisons of background characteristics and antibody titers.

\section{Statistical analysis}

We approximated antibody titer to a normal distribution by the Sinh-Arcsinh (SHASH) distribution, then performed univariate analysis using background, comorbidities, and laboratory data as explanatory variables. Using all variables with $p$ values of 0.2 or less in this analysis, we performed multivariable regression analysis (least square) by GraphPad Prism 9.

\section{Clinical course of HD patients with COVID-19 in unvaccinated and breakthrough infection}

Twenty-seven HD patients admitted to our hospital (25 patients and 2 patients belong to Tokyo Saiseikai Central hospital and Shimoochiai Clinic, respectively) with
COVID-19 from September 2020 to September 2021 were classified into the unvaccinated group $(n=15)$ and the twodose COVID-19 vaccine group $(n=12)$ (by BNT162b2 mRNA COVID-19 vaccine or the mRNA-1273 COVID-19 vaccine). Of vaccine group, 11 cases that met the definition of "breakthrough infection", were classified as breakthrough infection group $(n=11)$. Those patients were categorized the severity of COVID-19 into four levels from mild to critical, "Mild" as a case with various symptoms such as fever, cough, etc., but no shortness of breath or imaging abnormalities; "Moderate" as a case with evidence of lower respiratory tract disease on clinical or imaging evaluation and a $\mathrm{SpO}_{2}$ of $94 \%$ or higher on room air at sea level; "Severe" as a case with a $\mathrm{SpO}_{2}$ of less than $94 \%$ on room air at sea level and required oxygen therapy; "Critical" as a case in which more than $8 \mathrm{~L}$ of oxygen was needed to maintain $\mathrm{SpO}_{2}$ above $94 \%$, requiring a high-flow nasal cannula or intubation [7]. We compared the background characteristics, comorbidities, laboratory findings (CRP, LDH, KL-6 and ferritin), severity of COVID-19 and prognosis between unvaccinated group and breakthrough infection group.

\section{Clinical course of HD and non-HD patients with breakthrough infection}

Sixteen patients with breakthrough infection admitted to our hospital (15 patients and 1 patient belong to Tokyo Saiseikai Central hospital and Shimoochiai Clinic, respectively) between July and September 2021. We measured anti-S1 antibody titers of samples collected on admission day or on most recent pre-infection (if we had routine blood test) with the aim of minimizing the effect of infection on antibody titer. Samples of 12 out of 16 patients were collected within 2 weeks before to 3 days after the onset of COVID-19. We classified them into the HD group $(n=8)$ and non-HD group $(n=4)$ and performed between-group comparisons of the background characteristics, laboratory findings, antibody titer, and prognosis.

\section{SARS-CoV-2 antibody titer in patients with post-vaccine infections}

In the present study, we defined post-vaccine infection as cases that infected COVID-19 after at least one dose of vaccine. Twenty-three patients with post-vaccine infection admitted to our hospital (21 patients and 2 patients belong to Tokyo Saiseikai Central hospital and Shimoochiai Clinic, respectively) between July and September 2021. We measured anti-S1 antibody titers of samples collected on admission day or on most recent pre-infection (if we had routine blood test). Samples of 18 out of 23 patients were collected within 2 weeks before to 3 days after the onset of COVID-19. We classified them into the HD group $(n=9)$ 
and non-HD group ( $n=9)$, and plotted severity on the $X$-axis and antibody titer on the $Y$-axis.

Median values were compared using the Mann-Whitney $U$ test. Frequencies between groups were compared using Fisher's exact test or the Chi-square test. Statistical significance was set at $p<0.05$. All studies were conducted with informed consent and approved by the research ethics committee of the Tokyo Saiseikai Central Hospital (approval number: 2021-015-01).

\section{Results}

\section{SARS-CoV-2 antibody titers in the HD and control groups after vaccination}

The mean age of the HD group (70.4 years old) was significantly higher than the control group (40.3 years old), and there were significantly more males in the HD group (control: $17.1 \%$ vs. HD: $63.5 \%, p<0.01$ ) (Supplement Table 1). The antibody titers were significantly lower in the HD group $(n=104)$ than the healthy control group

Table 1 Univariate and multivariable analysis of factors affecting antibody titers among HD patients

\begin{tabular}{|c|c|c|c|c|c|c|c|}
\hline \multirow[t]{2}{*}{ Variable } & \multicolumn{3}{|l|}{ Univariate } & \multicolumn{4}{|l|}{ Multivariable } \\
\hline & $B$ coefficient & $95 \% \mathrm{CI}$ & $p$ value & $B$ coefficient & $95 \% \mathrm{CI}$ & VIF & $p$ value \\
\hline Smoke & -0.093 & -0.1634 to -0.02347 & 0.009 & -0.7864 & -1.527 to -0.04584 & 1.097 & 0.038 \\
\hline Duration of HD & 20.260 & 1.801 to 38.72 & 0.032 & 0.00414 & -0.001833 to 0.01011 & 1.163 & 0.168 \\
\hline Sex & 0.095 & 0.0004788 to 0.1886 & 0.049 & 0.1918 & -0.4730 to 0.8567 & 1.212 & 0.562 \\
\hline $\mathrm{HbA1c}$ & 0.342 & -0.01400 to 0.6981 & 0.059 & 0.1587 & -0.1004 to 0.4177 & 1.029 & 0.222 \\
\hline$\beta 2-\mathrm{MG}$ & -0.952 & -2.069 to 0.1657 & 0.094 & -0.02263 & -0.06955 to 0.02430 & 1.176 & 0.334 \\
\hline Age & -1.773 & -4.206 to 0.6599 & 0.151 & -0.03236 & -0.05719 to -0.007537 & 1.266 & 0.012 \\
\hline Cardiovascular disease & 0.050 & -0.02801 to 0.1271 & 0.208 & & & & \\
\hline $\mathrm{Hb}$ & 0.098 & -0.07785 to 0.2738 & 0.272 & & & & \\
\hline Plt & -0.609 & -1.821 to 0.6028 & 0.321 & & & & \\
\hline CK & 6.473 & -7.248 to 20.19 & 0.352 & & & & \\
\hline ALP & 2.748 & -3.440 to 8.935 & 0.381 & & & & \\
\hline $\mathrm{Cr}$ & 0.232 & -0.2944 to 0.7581 & 0.384 & & & & \\
\hline T-Bil & -0.010 & -0.03732 to 0.01665 & 0.449 & & & & \\
\hline Cerebral hemorrhage & -0.019 & -0.06955 to 0.03164 & 0.459 & & & & \\
\hline Glu & 2.552 & -5.837 to 10.94 & 0.548 & & & & \\
\hline TIBC & -3.150 & -14.11 to 7.814 & 0.570 & & & & \\
\hline Hypertension & 0.024 & -0.05993 to 0.1077 & 0.573 & & & & \\
\hline UN & -0.704 & -3.553 to 2.145 & 0.625 & & & & \\
\hline Ferittin & -3.329 & -17.47 to 10.81 & 0.642 & & & & \\
\hline AST & 0.769 & -2.501 to 4.040 & 0.642 & & & & \\
\hline Cerebtral infarction & 0.011 & -0.03655 to 0.05779 & 0.656 & & & & \\
\hline LDH & -1.620 & -10.01 to 6.775 & 0.703 & & & & \\
\hline CRP & -0.021 & -0.1780 to 0.1362 & 0.792 & & & & \\
\hline PTHi & -2.290 & -25.81 to 21.23 & 0.847 & & & & \\
\hline Amylase & -1.032 & -12.09 to 10.03 & 0.854 & & & & \\
\hline $\mathrm{Alb}$ & -0.005 & -0.06864 to 0.05766 & 0.863 & & & & \\
\hline ALT & 0.184 & -1.998 to 2.367 & 0.867 & & & & \\
\hline $\mathrm{TP}$ & -0.008 & -0.1174 to 0.1005 & 0.878 & & & & \\
\hline Diabetes mellitus & 0.007 & -0.09230 to 0.1068 & 0.885 & & & & \\
\hline WBC & -8.842 & -383.2 to 365.5 & 0.963 & & & & \\
\hline UA & -0.003 & -0.2644 to 0.2593 & 0.985 & & & & \\
\hline
\end{tabular}

$C I$ confidence interval, $V I F$ variance inflation factor, $H D$ hemodialysis, $H b$ hemoglobin, $\beta 2-M G \beta 2$-microglobulin, $P l t$ platelet, $C K$ creatinine kinase, $A L P$ alkaline phosphatase, $C r$ creatinine, $T$-Bil total-bilirubin, $G l u$ glucose, $T I B C$ total iron binding capacity, $U N$ urea nitrogen, $A S T$ aspartate aminotransferase, $L D H$ lactate dehydrogenase, $C R P$ C-reactive protein, $P T H i$ intact parathyroid hormone, $A l b$ albumin, $A L T$ alanine aminotransferase, $T P$ total protein, $W B C$ white blood cell, $U A$ uric acid 
Fig. 1 SARS-CoV-2 antibody titer in HD and control group after full vaccination. The antibody titers were significantly lower in the HD group $(n=104)$ than in the healthy control group $(n=35)$ after full vaccination (control: $12,722.2$ vs HD: $3848.8 \mathrm{AU} / \mathrm{mL}, p<0.001)$ (A). When we conducted age-matched analysis (control: 8193.3 vs HD: $4295.8 \mathrm{AU} / \mathrm{mL}$, $p=0.005)(\mathbf{B})$ and sex-matched analysis (only males; control: 9998.0 vs HD: $3650.2 \mathrm{AU} / \mathrm{mL}$, $p<0.001(\mathbf{C})$, only females; control: $13,285.8$ vs HD: 4193.8 AU/mL, $p<0.001$ (D)), the antibody titers were still significantly lower in HD group in every analysis. $H D$ hemodialysis
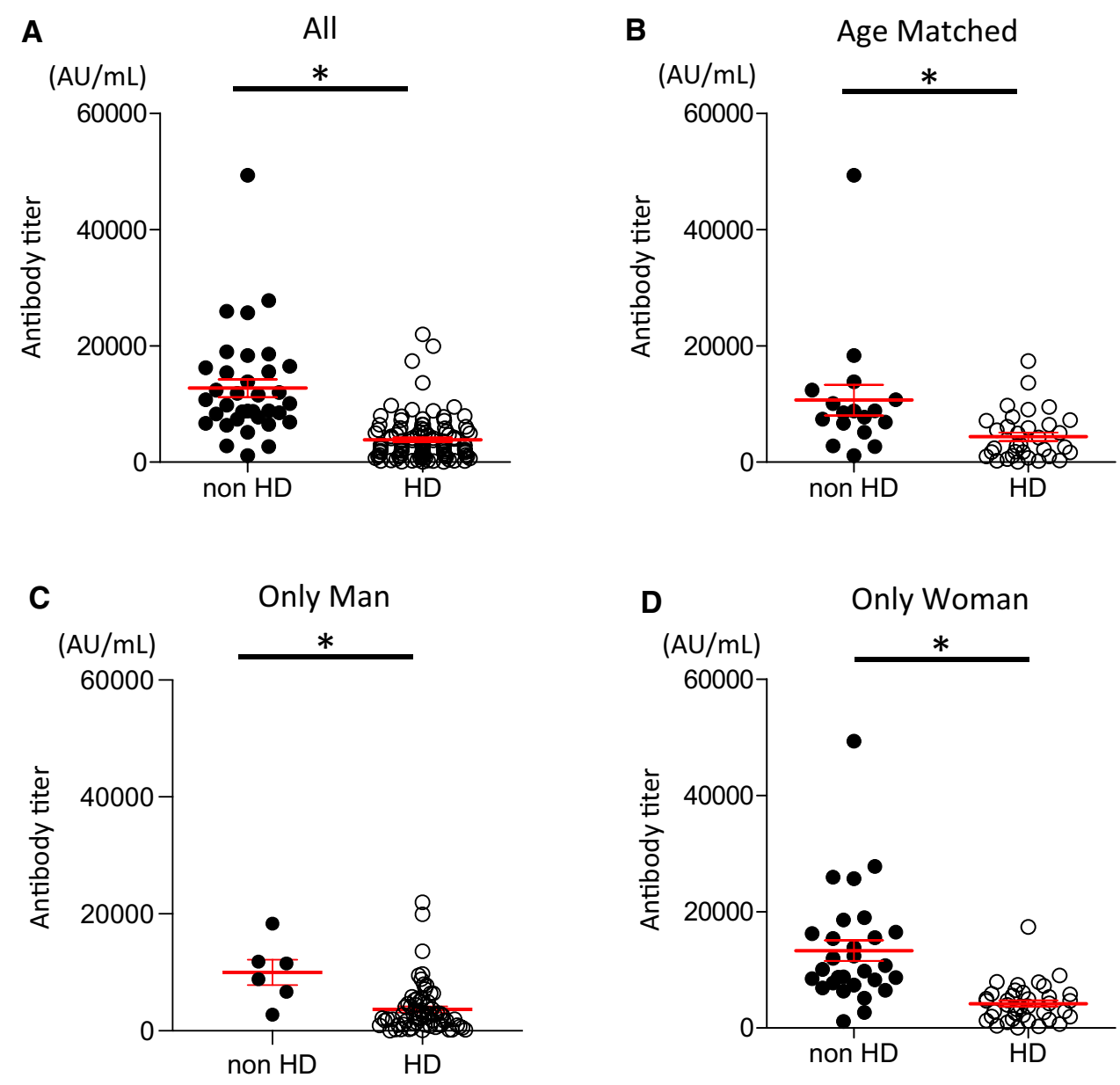

$(n=35)$ (control: 12,722.2 AU/mL vs HD: $3848.8 \mathrm{AU} / \mathrm{mL}$, $p<0.001$ ) (Fig. 1A). In an age-matched analysis (comparison of patients between 43 years of age (minimum age of HD group) and 60 years of age (mean age; control $[n=13]$ 50.5 years old vs. HD $[n=26] 53.5$ years), the antibody titers were still significantly lower in the HD group (control: 8193.3 AU/mL vs HD: 4295.8 AU/mL, $p=0.005$ ) (Fig. 1B). Further, on conducting a sex-matched analysis, the antibody titers were still significantly lower in HD group both in only males (control: 9998.0 AU/mL vs HD: 3650.2 AU/mL, $p<0.001$ ) and in only females (control: 13,285.8 AU/mL vs HD: $4193.8 \mathrm{AU} / \mathrm{mL}, p<0.001$ ) (Fig. 1C, D).

\section{Statistical analysis}

In the HD group, the antibody titer was approximated to a normal distribution by the SHASH distribution (Shapiro-Wilk test: $p>0.05$ ), then performed univariate analysis using background, comorbidities, and laboratory data as explanatory variables. As a result, age, sex, duration of HD, $\beta 2-M i c r o g l o b u l i n, H b A 1 c$, and smoking history were variables with $p$ values of 0.2 or less in this analysis (Table 1). Using these variables as explanatory variable, we performed multivariable regression analysis, resulted in only age and smoking history were significantly independent factors associated with antibody titer (Table 1). A plot of the correlation between age and antibody titer showed a negative correlation trend (Fig. 2A). Also, smoking groups $(n=13)$ had significantly lower antibody titers than non-smoking group $(n=84)$ $(p=0.007)$ (Fig. 2B).

\section{Clinical course of HD patients with COVID-19 in unvaccinated and breakthrough infection}

Among HD patients infected with COVID-19, there was no significant difference in background characteristics and comorbidities between the unvaccinated group (UV) $(n=15)$ and the breakthrough infection group (BI) $(n=11)$ (Table 2). We evaluated CRP, LDH, KL-6, and ferritin levels on admission and CRP, LDH, and ferritin levels at the maximum during hospitalization to compare the clinical course between the two groups. There were no significant differences on admission except for KL-6 (UV; $351.2 \mathrm{U} / \mathrm{mL}$ vs BI; $235.9 \mathrm{U} / \mathrm{mL}, p=0.02$ ). However, at the maximum during hospitalization, LDH (UV; $476.3 \mathrm{U} / \mathrm{L}$ vs $\mathrm{BI} ; 303.8 \mathrm{U} / \mathrm{L}, p=0.01)$ was significantly lower in the 
Fig. 2 Independent factors associated with antibody titers in HD patients. Multivariable regression analysis was performed by using age, sex, duration of $\mathrm{HD}, \beta 2$-microglobulin, HbA1c, and smoke as explanatory variables. As a result, only age and smoke were significantly independent factors associated with antibody titer. There was a non-significant negative correlation between age and antibody titer (A). Antibody titers were significantly lower in smoker than in non-smoker $(p=0.007)(\mathbf{B})$
A

Age

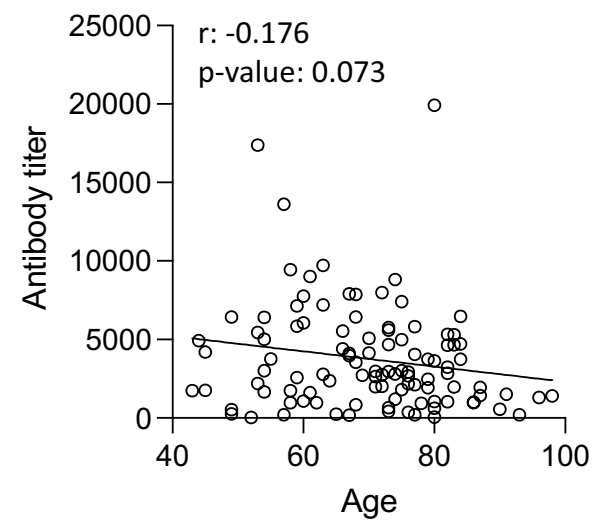

B

Smoke

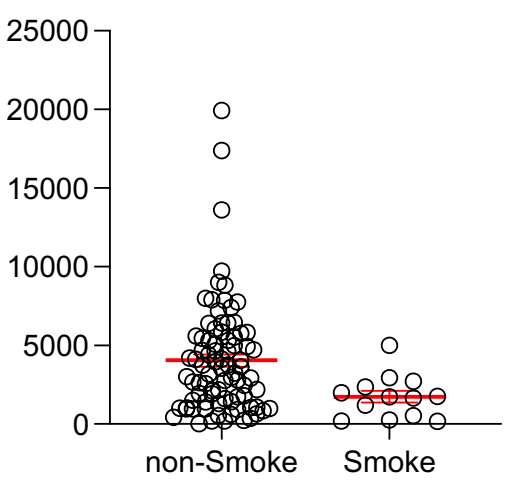

breakthrough infection group. Further, CRP and ferritin levels tended to be more exacerbated during hospitalization in the unvaccinated group than the breakthrough infection group (Table 2, Fig. 3). The severity of COVID$19(p=0.02)$ and the number of admission days (UV; 28.7 days vs BI; 13.1 days, $p<0.01$ ) was significantly lower in the breakthrough infection group. Four patients died in the unvaccinated group, however, all patients in the breakthrough infection group recovered completely (Table 2).

\section{Clinical course of HD and non-HD patients with breakthrough infection}

There were no significant differences in background (age, sex, comorbidity) between the HD and non-HD groups. The antibody titer was not significantly different between both groups (HD; 1758.9 AU/mL vs non-HD; $1374.4 \mathrm{AU} /$ $\mathrm{mL}$ ). The outcome of breakthrough infection, COVID-19 severity and the number of admission days (HD; 12.0 days vs non-HD; 8.5 days) was not significantly different between both groups. Although there was one death in the non-HD group (patient with stage IV pancreatic cancer), all other patients in both groups recovered completely (Table 3).

\section{SARS-CoV-2 antibody titer in patients with post-vaccine infections}

When we plotted severity on the $X$-axis and antibody titer on the $Y$-axis, severity tended to be higher with lower antibody titer in non-HD patients. However, in HD patients, there were cases of moderate or severe even with antibody titers around $4000 \mathrm{AU} / \mathrm{mL}$, while all mild cases had antibody titers under $1000 \mathrm{AU} / \mathrm{mL}$ (Fig. 4).

\section{Discussion}

Our study revealed that HD patients had significantly lower antibody titer than the control, and age and smoking history were identified as significant independent factors associated with antibody titer. We also clarified that vaccine improved severity of COVID-19 and hospitalization period of HD patients with breakthrough infection. All HD patients with breakthrough infection had antibody titer lower than $4160 \mathrm{AU} / \mathrm{mL}$ : the known threshold for neutralization in vitro ( 0.95 probability of obtaining PRNT (plaque reduction neutralization test) ID50 at a 1:250 dilution). However, they had significantly better laboratory findings, severity of COVID-19, and hospitalization period than the unvaccinated HD patients with COVID-19. Among patients with post-vaccine infections, some cases with high antibody titers became severe clinical course in HD patients, whereas COVID-19 severity tended to be higher with lower antibody titer in nonHD patients.

The $\mathrm{S} 1$ subunit is a component of the spike protein, which is one of the major structural proteins of SARS-CoV-2 mediating cell surface binding via the receptor-binding domain [6]. We used Abbott SARS-CoV-2 IgG II Quantitative Antibody Assay to detect anti-S1 IgG antibodies which strongly correlated with neutralization $[4,5]$. It has been reported that anti-S1 antibody titer was significantly lower in HD patients, particularly those aged over 60-75 years, than in healthy controls $[8,9]$. Our results also showed significantly lower antibody titers in the HD group than the control group (Fig. 1).

It remains to be clarified why antibody titers to the COVID-19 vaccine were reduced in HD patients. Hormonal responses to influenza and hepatitis B virus vaccines have been reported to be significantly reduced [6]. This is because both the innate and adaptive immune systems are disturbed by uremia, leading to decreased antigen processing and reduced cell-mediated and antibody-mediated 
Table 2 Clinical course of HD patients with COVID-19 in unvaccinated and breakthrough infection

\begin{tabular}{|c|c|c|c|}
\hline & $\mathrm{UV}(n=15)$ & $\mathrm{BI}(n=11)$ & $p$ value \\
\hline \multicolumn{4}{|l|}{ Background } \\
\hline Age (year-old) & 65.9 & 68.3 & 0.671 \\
\hline Male $(n)$ & 10 & 8 & $>0.999$ \\
\hline \multicolumn{4}{|l|}{ Comorbidities (n) } \\
\hline Diabetes & 9 & 6 & $>0.999$ \\
\hline Hypertension & 12 & 6 & 0.218 \\
\hline Hyperlipidemia & 4 & 4 & 0.683 \\
\hline Malignancy & 3 & 2 & $>0.999$ \\
\hline Cardiovascular disease & 8 & 6 & $>0.999$ \\
\hline \multicolumn{4}{|l|}{ COVID-19 } \\
\hline Duration of HD (years) & 6.7 & 7.6 & 0.941 \\
\hline \multicolumn{4}{|l|}{ Treatment $(n)$} \\
\hline FPV & 11 & 0 & \\
\hline RDV & 4 & 9 & \\
\hline $\mathrm{TCZ}+$ Pulse & 4 & 2 & \\
\hline $\mathrm{C} / \mathrm{I}$ & 1 & 6 & \\
\hline None & 0 & 2 & \\
\hline \multicolumn{4}{|l|}{ Severity $(n)$} \\
\hline Mild & 0 & 2 & 0.020 \\
\hline Moderate & 0 & 3 & \\
\hline Severe & 8 & 5 & \\
\hline Critical & 7 & 1 & \\
\hline Admission days (days) & 28.7 & 13.1 & 0.001 \\
\hline \multicolumn{4}{|l|}{ Outcome $(n)$} \\
\hline Survive & 11 & 11 & 0.113 \\
\hline Death & 4 & 0 & \\
\hline \multicolumn{4}{|l|}{ Laboratory data } \\
\hline \multicolumn{4}{|l|}{ On admission } \\
\hline $\mathrm{CRP}(\mathrm{mg} / \mathrm{dL})$ & 10.5 & 6.0 & 0.226 \\
\hline LDH (U/L) & 319.9 & 268.2 & 0.291 \\
\hline Ferritin (ng/mL) & 729.2 & 303.2 & 0.131 \\
\hline KL-6 (U/mL) & 351.2 & 235.9 & 0.015 \\
\hline \multicolumn{4}{|c|}{ Maximum during hospitalization } \\
\hline CRP (mg/dL) & 13.9 & 7.3 & 0.064 \\
\hline LDH (U/L) & 476.3 & 303.8 & 0.009 \\
\hline Ferritin $(\mathrm{ng} / \mathrm{mL})$ & 871.5 & 377.5 & 0.086 \\
\hline
\end{tabular}

$U V$ unvaccinated, BI breakthrough infection, COVID-19 coronavirus disease 2019, FPV favipiravir, $R D V$ remdesivir, $T C Z$ tocilizumab $(8 \mathrm{mg} / \mathrm{kg})$, Pulse methylprednisolone pulse (3 days of 500 and $1000 \mathrm{mg} /$ day for $<75$ and $>75 \mathrm{~kg}$, respectively), $C / I$ casirivimab and imdevimab

immune responses in HD patients [8]. Therefore, the low vaccine antibody titer of COVID-19 is likely to be caused by dysfunction of both innate and adaptive immunity in HD patients. Humoral and B-cell responses against the COVID19 vaccine were markedly impaired in HD patients compared to controls. Additionally, although most antigen-specific B cells in the control group were identified in the plasmablast or post-switch memory B cells, they were identified among pre-switch and naïve B cells in HD patients [10]. Among HD patients, characteristics of patients with particularly low antibody titers have been investigated. Older age, BMI under 30 , low albumin level, and low intravenous iron dose were identified as factors affecting antibody titer by multivariable analysis [3]. In our study, only age and smoking history were significantly independent factors associated with antibody titer (Table 1). In addition, smoking groups had significantly lower antibody titers than non-smoking group (Fig. 2B). Smoking is associated with a dysfunctional immune system, and it was previously reported that antibody titers following influenza vaccination decline more rapidly in smokers [11]. It is assumed that decreased immune function due to old age and smoking habit led to low antibody titer. As high and repeated doses of hepatitis B vaccine are recommended for HD patients [12], and the third dose of COVID-19 vaccine enhances humoral response, especially in those with lower antibody titers after the second dose [13], a third dose of the COVID-19 vaccine should also be considered in HD patients, especially elderly smoking patients.

Over 10,000 SARS-CoV-2 vaccine breakthrough infections have been reported in the United States, and $10 \%$ of patients are hospitalized [14]. In the general population, the rate of severe complications or death among the vaccinated group was threefold lower than that of matched unvaccinated controls, and age is the most important factor affecting outcomes of breakthrough infection [15]. Furthermore, vaccinated HD patients had lower rates of infection and significantly higher survival rates than unvaccinated controls [16]. In the present study, the severity of COVID19 , and number of admission days were significantly lower in the breakthrough infection group than the unvaccinated group among HD patients (Table 2). These results indicates that the vaccine prevented severe complication after breakthrough infection. Clinical symptoms of COVID-19 worsen around 7-10 days from onset due to systemic hyperinflammation caused by uncontrolled cytokine overproduction. To prevent this cytokine overproduction, the rapid reduction of viral load by the early antiviral response is most important [17]. Our study showed that, LDH level, which was not significantly different on admission day, was significantly lower in the breakthrough infection group at the maximum level during hospitalization. In addition, CRP and ferritin levels during hospitalization tended to be less exacerbated in the breakthrough infection group (Table 2, Fig. 3), implying that the early antiviral response activated by the vaccine prevented the cytokine overproduction. However, it is possible that the differences in treatment affected on the difference in prognosis between the two groups. Since remdesivir has been reported to significantly better prognosis compared to favipiravir [18], the low rate of remdesivir administration may have had negative impact on prognosis in unvaccinated 
Fig. 3 HD patients with COVID-19 in unvaccinated and breakthrough infection. We compared KL-6 on admission and CRP, LDH, and ferritin at the maximum during hospitalization between unvaccinated (UV) and breakthrough infection (BI) among HD patients admitted with COVID-19. KL-6 (UV; $351.2 \mathrm{U} / \mathrm{mL}$ vs $\mathrm{V} ; 235.9 \mathrm{U} / \mathrm{mL}, p=0.02)(\mathbf{A})$ and LDH (UV; $476.3 \mathrm{U} / \mathrm{L}$ vs $\mathrm{V} ; 303.8 \mathrm{U} / \mathrm{L}, p=0.01)(\mathbf{B})$ were significantly lower in the breakthrough infection group. Also, CRP (UV; $13.9 \mathrm{mg} / \mathrm{dL}$ vs $\mathrm{V} ; 7.3 \mathrm{mg} / \mathrm{dL}, p>0.05)(\mathbf{C})$ and ferritin (UV; $871.5 \mathrm{ng} / \mathrm{mL}$ vs $\mathrm{V} ; 377.5 \mathrm{ng} / \mathrm{mL}, p>0.05)$ (D) tended to be lower in the breakthrough infection group
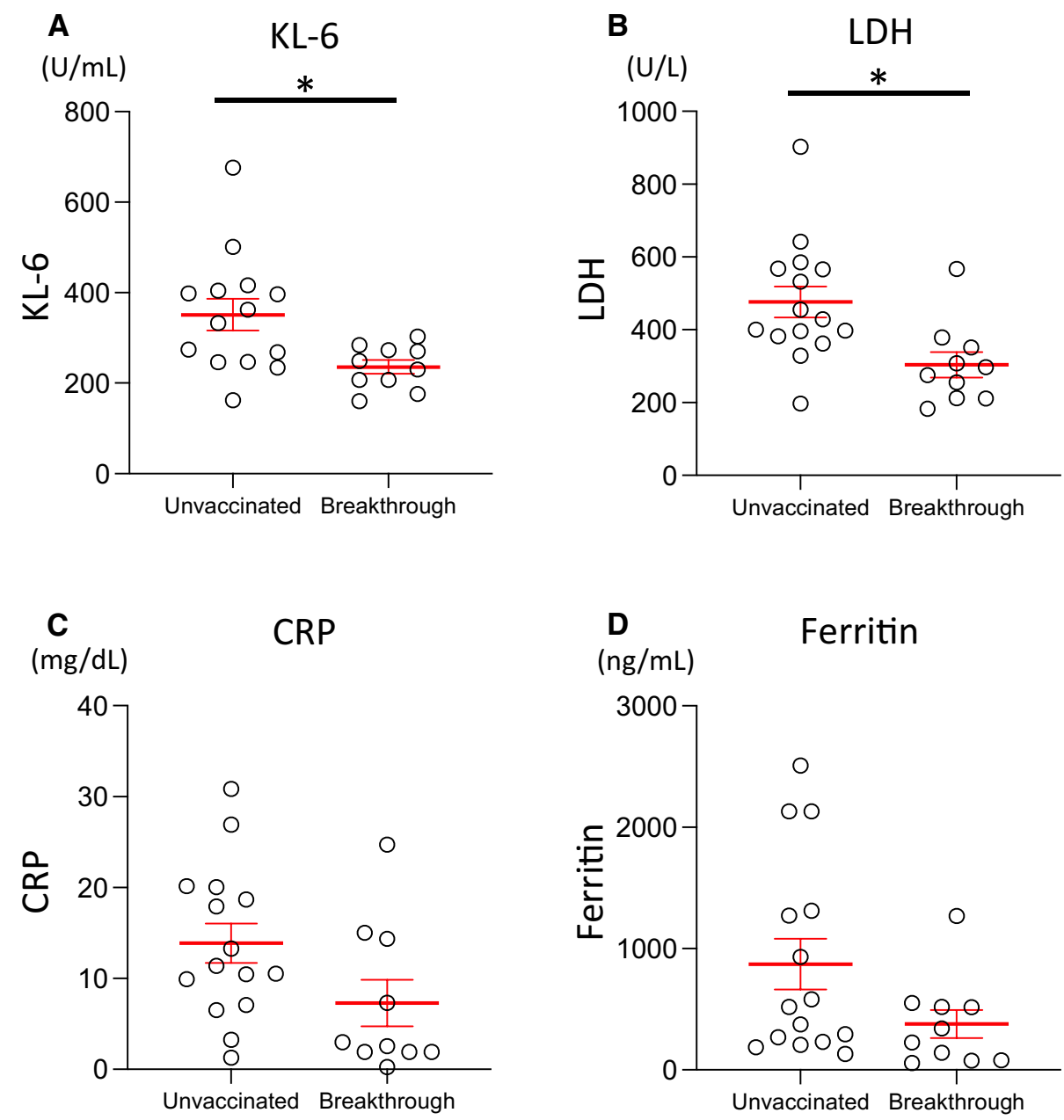

group. In addition, among cases with moderate or higher, 6 out of 9 patients in the breakthrough infection group, while only 1 out of 15 patients in the unvaccinated group were administered Casirivimab/Imdevimab. Furthermore, 3 out of 7 patients with critical in the unvaccinated group did not receive TCZ and steroid pulse therapy (2 of 3 patients died). Even with the above possibilities, we still think that the vaccine prevented the severe complications of COVID-19.

While developing antibodies through a vaccine is important, it is still unclear whether the antibody titer itself causes clinical differences. In vitro, anti-S1 antibody above $4160 \mathrm{AU} / \mathrm{mL}$ is the threshold corresponding to a 0.95 probability of obtaining a PRNT ID50 at a 1:250 dilution [19]. Further, higher antibody titers were associated with both a lower risk of breakthrough infection and lower viral RNA copy number [5]. However, there are no generally accepted clinical cutoff values for antibodies to protect against breakthrough infections or to prevent severe disease. All patients with breakthrough infection in HD patients and non-HD patients had antibody titers of less than $4160 \mathrm{AU} / \mathrm{mL}$ (Supplement Table 3). In these cases, there were no fatal cases regardless of antibody titers, except for one case in the non-HD group, suggesting that the vaccine could prevent severe disease to some extent. In addition, there was no significant difference in COVID-19 severity or and the hospitalization period between the HD and non-HD patients with breakthrough infection (Table 3). Among patients with post-vaccine infection, some HD patients became severely to critically ill even with antibody levels of about 2000-4000 AU/mL. On the other hand, in non-HD patients, those who became severely to critically ill had antibody levels of less than $200 \mathrm{AU} / \mathrm{mL}$. Therefore, we compared these two groups to investigate the difference in antibody titer and disease severity. Non-HD patients tended to have a higher severity of disease with lower antibody titers (Fig. 4A). Of the four non-HD patients with higher severity, two had received only one dose of vaccine, and the other two had chronic lymphocytic leukemia and stage IV pancreatic cancer, respectively (Supplement Table 3). It is unclear whether the reason for the low antibody levels in these patients was due to the underlying disease, but these immunocompromised patients require sufficiently high antibody levels to prevent severe disease. On the other hand, in HD patients, 


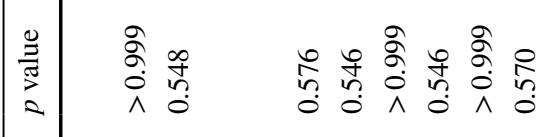

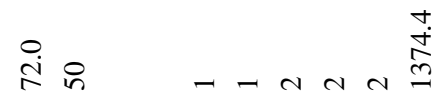

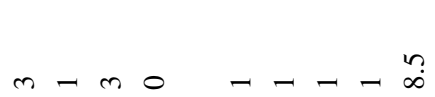

n-

ma

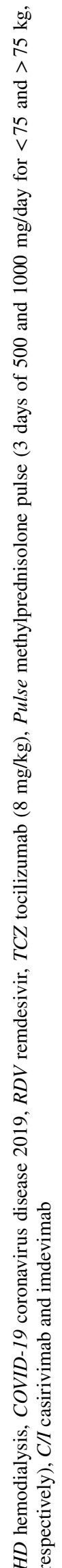

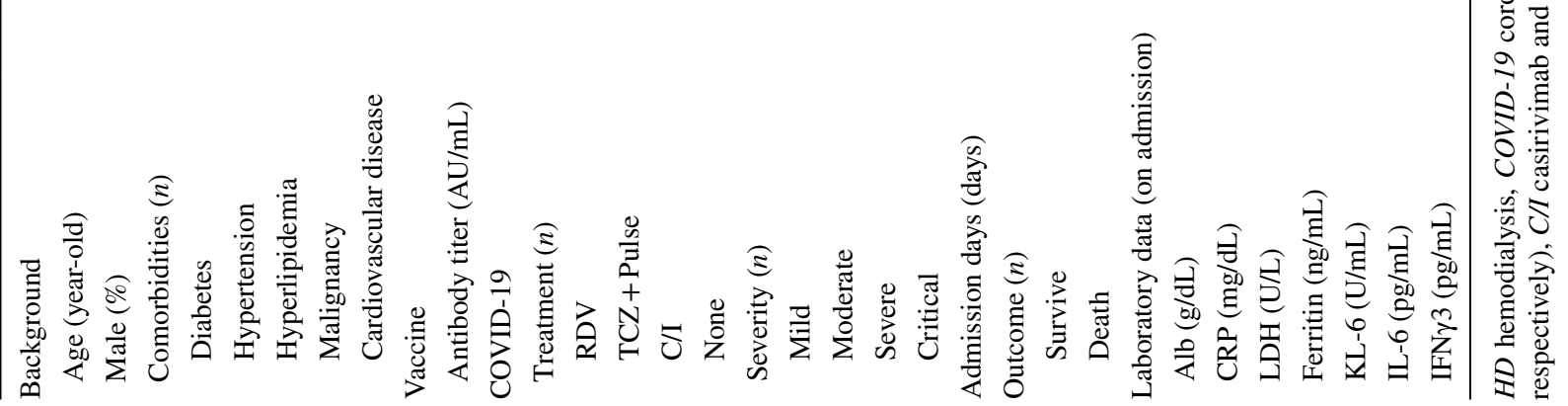




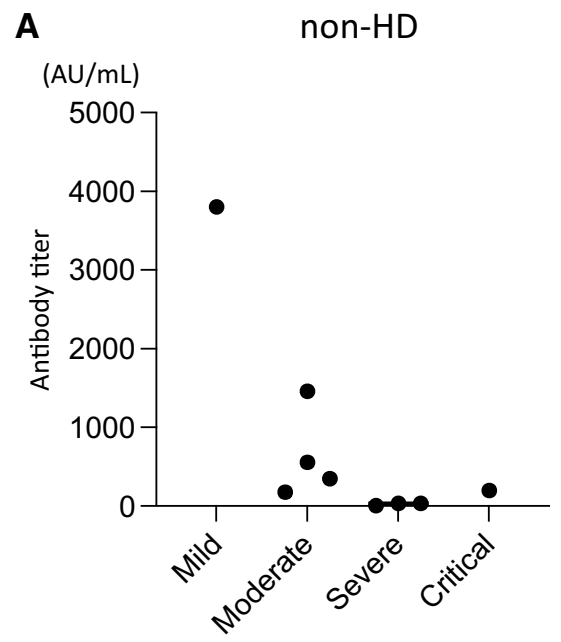

Fig. 4 Association between severity and antibody titer in post-vaccine infection. To investigate the relationship between the antibody titer and the prognosis, we plotted the severity of COVID-19 on the $X$-axis and antibody titer on the $Y$-axis in patients admitted with COVID-19 after at least one dose of COVID-19 vaccine. The sever-

there were cases of moderate or severe even with antibody titers around $4000 \mathrm{AU} / \mathrm{mL}$ (Fig. 4B), suggesting that due to impaired immunity, much higher antibody titers were required for the prevention of onset and severity in HD patients. According to these results, a third vaccination should be considered for immunocompromised patients in the appropriate time.

Important limitations of our study include the small number of patients with breakthrough infections. Additionally, we measured anti-S1 antibody titers to investigate the effect of the vaccine on humoral immunity, but have not been able to examine neutralization titers or cellular immunity. Further, although we only applied samples collected within 2 weeks before to 3 days after the onset of COVID19 , we could not unify the measurement time after the onset of COVID-19. The antibody titer did not rise within 3 days after onset in past report [20] and samples would be properly preserved within 2 weeks, The number of breakthrough infections is expected to increase; therefore, a study with a larger number of patients is required in the future.

\section{Conclusion}

The COVID-19 vaccine improved the severity of COVID19 and hospitalization period of breakthrough infection in HD patients, although they, especially in elderly smokers obtain lower antibody titers than healthy controls. There was no significant association between antibody titer and severity in HD patients.

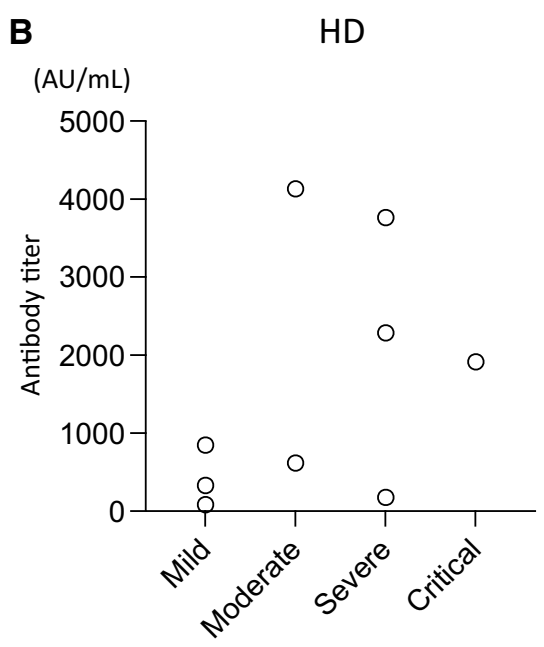

ity tended to be higher with lower antibody titer in non-HD patients. However, in HD patients, there were two cases of moderate or severe even with antibody titers around $4000 \mathrm{AU} / \mathrm{mL}$, while all mild cases had antibody titers under $1000 \mathrm{AU} / \mathrm{mL}$. HD hemodialysis

Supplementary Information The online version contains supplementary material available at https://doi.org/10.1007/s10157-022-02188-y.

Author contributions AY, KK and MR designed for this study. MT, $\mathrm{AY}$, and KK collected the clinical information. MT and AY analyzed the data and drafted the manuscript. All authors revised the paper, and all authors approved the final version of the manuscript.

Funding No funding or sponsorship was received for this study or publication of this article.

Data availability The datasets generated during and/or analyzed during the current study are available from the corresponding author on reasonable request.

\section{Declarations}

Conflict of interest All the authors have declared no competing interest.

Ethical approval The research ethics committee of the Tokyo Saiseikai Central Hospital evaluated and approved the study protocol (approval number 2021-015-01).

Informed consent All patients gave oral or written consent to have antibody titers measured.

Authorship All named authors meet the International Committee of Medical Journal Editors (ICMJE) criteria for authorship for this article, take responsibility for the integrity of the work, and have given their approval for this version to be published. 


\section{References}

1. Polack FP, Thomas SJ, Kitchin N, Absalon J, Gurtman A, Lockhart S, et al. Safety and efficacy of the BNT162b2 mRNA covid19 vaccine. N Engl J Med. 2020;383(27):2603-15. https://doi.org/ 10.1056/NEJMoa2034577.

2. Frantzen L, Cavaille G, Thibeaut S, El-Haik Y. Efficacy of the BNT162b2 mRNA covid-19 vaccine in a hemodialysis cohort. Nephrol Dial Transplant. 2021. https://doi.org/10.1093/ndt/gfab1 65.

3. Yen CC, Lin SY, Chen SC, Chiu YW, Chang JM, Hwang SJ. COVID-19 vaccines in patients with maintenance hemodialysis. J Pers Med. 2021. https://doi.org/10.3390/jpm11080789.

4. Garcia-Beltran WF, Lam EC, Astudillo MG, Yang D, Miller TE, Feldman J, et al. COVID-19-neutralizing antibodies predict disease severity and survival. Cell. 2021;184(2):476-88.e11. https:// doi.org/10.1016/j.cell.2020.12.015.

5. Bergwerk M, Gonen T, Lustig Y, Amit S, Lipsitch M, Cohen C, et al. Covid-19 breakthrough infections in vaccinated health care workers. N Engl J Med. 2021. https://doi.org/10.1056/NEJMo a2109072.

6. Speer C, Göth D, Benning L, Buylaert M, Schaier M, Grenz $\mathrm{J}$, et al. Early humoral responses of hemodialysis patients after COVID-19 vaccination with BNT162b2. Clin J Am Soc Nephrol. 2021;16(7):1073-82. https://doi.org/10.2215/cjn.03700321.

7. Coronavirus Disease 2019 (COVID-19) treatment guidelines. https://www.covid19treatmentguidelines.nih.gov/. Accessed 28 Nov 2021

8. Jahn M, Korth J, Dorsch O, Anastasiou OE, Sorge-Hädicke B, Tyczynski B, et al. Humoral response to SARS-CoV-2-vaccination with BNT162b2 (Pfizer-BioNTech) in patients on hemodialysis. Vaccines (Basel). 2021. https://doi.org/10.3390/vaccines9040360.

9. Yanay NB, Freiman S, Shapira M, Wishahi S, Hamze M, Elhaj M, et al. Experience with SARS-CoV-2 BNT162b2 mRNA vaccine in dialysis patients. Kidney Int. 2021;99(6):1496-8. https://doi. org/10.1016/j.kint.2021.04.006.

10. Rincon-Arevalo H, Choi M, Stefanski AL, Halleck F, Weber U, Szelinski F, et al. Impaired humoral immunity to SARS-CoV-2 BNT162b2 vaccine in kidney transplant recipients and dialysis patients. Sci Immunol. 2021. https://doi.org/10.1126/sciimmunol. abj1031.

11. Watanabe M, Balena A, Tuccinardi D, Tozzi R, Risi R, Masi D, et al. Central obesity, smoking habit, and hypertension are associated with lower antibody titres in response to COVID-19 mRNA vaccine. Diabetes Metab Res Rev. 2021. https://doi.org/10.1002/ dmrr.3465.
12. Goupil R, Benlarbi M, Beaubien-Souligny W, Nadeau-Fredette AC, Chatterjee D, Goyette G, et al. Short-term antibody response after 1 dose of BNT162b2 vaccine in patients receiving hemodialysis. CMAJ. 2021;193(22):E793-e800. https://doi.org/10.1503/ cmaj. 210673.

13. Ducloux D, Colladant M, Chabannes M, Yannaraki M, Courivaud C. Humoral response after 3 doses of the BNT162b2 mRNA COVID-19 vaccine in patients on hemodialysis. Kidney Int. 2021;100(3):702-4. https://doi.org/10.1016/j.kint.2021.06.025.

14. CDC COVID-19 Vaccine Breakthrough Case Investigations Team. COVID-19 vaccine breakthrough infections reported to CDCUnite States, January 1-April 30, 2021. MMWR Morb Mortal Wkly Rep. 2021;70(21):792-3.

15. Butt AA, Nafady-Hego H, Chemaitelly H, Abou-Samra AB, Khal AA, Coyle PV, et al. Outcomes among patients with breakthrough SARS-CoV-2 infection after vaccination. Int J Infect Dis. 2021;110:353-8. https://doi.org/10.1016/j.ijid.2021.08.008.

16. Cserep G, Morrow D, Latchford K, Jesset R, Dosa A, Kirmizis D. The effect of a single dose of BNT162b2 vaccine on the incidence of severe COVID-19 infection in patients on chronic hemodialysis: a single-centre study. Clin Exp Nephrol. 2021. https://doi.org/ 10.1007/s10157-021-02118-4.

17. Jamilloux Y, Henry T, Belot A, Viel S, Fauter M, El Jammal $\mathrm{T}$, et al. Should we stimulate or suppress immune responses in COVID-19? Cytokine and anti-cytokine interventions. Autoimmun Rev. 2020;19(7): 102567. https://doi.org/10.1016/j.autrev. 2020.102567.

18. Kikuchi K, Nangaku M, Ryuzaki M, Yamakawa T, Yoshihiro O, Hanafusa N, et al. Survival and predictive factors in dialysis patients with COVID-19 in Japan: a nationwide cohort study. Ren Replace Ther. 2021;7(1):59. https://doi.org/10.1186/ s41100-021-00378-0.

19. Ebinger JE, Fert-Bober J, Printsev I, Wu M, Sun N, Prostko JC, et al. Antibody responses to the BNT162b2 mRNA vaccine in individuals previously infected with SARS-CoV-2. Nat Med. 2021;27(6):981-4. https://doi.org/10.1038/s41591-021-01325-6.

20. Zhao J, Yuan Q, Wang H, Liu W, Liao X, Su Y, et al. Antibody responses to SARS-CoV-2 in patients with novel coronavirus disease 2019. Clin Infect Dis. 2020;71(16):2027-34. https://doi.org/ 10.1093/cid/ciaa344.

Publisher's Note Springer Nature remains neutral with regard to jurisdictional claims in published maps and institutional affiliations. 